\title{
THE MAXIT AND MINIT OF A RING
}

\author{
by R. E. PEINADO and W. G. LEAVITT
}

(Received 23 September, 1964)

1. Introduction. In a recent paper [2] one of the authors has introduced the concept of module type of a ring, for rings with unit. The object of this paper is to generalize this concept to arbitrary rings, without assuming the existence of a unit. This is easily accomplished for rings with one-sided unit, and we shall define the type of such a ring. Theorem 2.5 gives a relation between this type and the module type of [2], and permits the immediate extension of all results in [2] to rings with one-sided unit.

We then define the maximal image type of a ring (to be called the maxit) as the l.u.b. of the types of those images of the ring having right units. Dually, the minimal image type (the minit) is the g.l.b. of the types of such images. Several of the properties of the maxit and minit are established, such as the fact that for rings with unit the module type and the maxit coincide.

All modules over a ring $A$ are assumed to be left $A$-modules, and are assumed to have a finite basis (if they have any at all).

2. The type of a ring. An $A$-module $M$ is defined to be a finitely based module if it contains a finite set $\left\{\alpha_{i}\right\}$ in terms of which each $\alpha \in M$ is uniquely representable.

THEOREM 2.1. A ring $A$ admits a finitely based module $M$ if and only if it has right units.

Proof. Let $M$ be a finitely based $A$-module and $\left\{\alpha_{i}\right\}(i=1, \ldots, n)$ a basis for $M$. Then for any basis element $\alpha_{j}$,

For $0 \neq a \in A$, we have

$$
\alpha_{j}=\sum_{i=1}^{n} x_{i} \alpha_{i} \quad\left(x_{i} \in A\right)
$$

$$
a \alpha_{j}=\sum_{i=1}^{n} a x_{i} \alpha_{i} .
$$

Since $\left\{\alpha_{i}\right\}$ is a basis, $a x_{j}=a$ and $a x_{i}=0(i \neq j)$. Thus $x_{j}$ is a right unit and the $x_{i}(i \neq j)$ are right annihilators of $A$. Conversely, if $A$ has right units, it clearly admits a finitely based $A$-module, namely the ring $A$ as a module over itself.

We shall say that a module $M$ has dimension $n$ if all its bases have $n$ elements. Call such a module dimensional. A ring $A$ is said to be dimensional if all finitely based modules over $A$ are dimensional.

THEOREM 2.2. For any non-dimensional ring $A$, with right units, there exist positive integers $(n, k)$ such that (i) any finitely based module, with basis of length $<n$, has dimension; (ii) for any finitely based module $M$ with basis of length $\geqq n$, there exists an integer $h$, with $n \leqq h<n+k$, such that $M$ has a basis of length $r$ if and only if $r=h+m k$, with $m \geqq 0$. Moreover such a module exists for arbitrary $h$.

Proof. This follows with small modifications the proof of [2, Theorem 1, p. 114]. 
Define the type of a ring $A$ with right units (designated $t(A)$ ) as follows: $t(A)=d$ if $A$ is dimensional; $t(A)=(n, k)$ as defined in Theorem 2.2 if $A$ is nondimensional; $t(A)=0$ if $A$ is the zero ring. We remark that a dual right type could have been defined using right modules. A ring with both left and right types would necessarily have a two-sided unit, and so would have a module type (as in [2]).

The matrix criterion of $[2$, p. 115] becomes in our context

THEOREM 2.3. A necessary and sufficient condition for the existence of an A-module with bases of length $n$ and $n+k$ is that there exist $n$ by $n+k$ and $n+k$ by $n$ matrices $B$ and $C$ over $A$ such that

$$
X B C=X \text { and } Y C B=Y
$$

for arbitrary vectors $X$ and $Y$ (of appropriate dimension) over $A$. Then

Proof. Let $\left\{\alpha_{i}\right\}(i=1, \ldots, n)$ and $\left\{\beta_{j}\right\}(j=1, \ldots, n+k)$ be bases for an $A$-module $M$.

$$
\alpha_{i}=\sum_{j=1}^{n+k} b_{i j} \beta_{j} \text { and } \beta_{i}=\sum_{j=1}^{n} c_{i j} \alpha_{j}
$$

Thus from the uniqueness of the representation of $M$ in terms of the bases it follows that

and

$$
x \sum_{r=1}^{n+k} b_{i r} c_{r j}= \begin{cases}x & \text { if } i=j \\ 0 & \text { if } i \neq j\end{cases}
$$

$$
y \sum_{s=1}^{n} c_{i s} b_{s j}= \begin{cases}y & \text { if } i=j, \\ 0 & \text { if } i \neq j,\end{cases}
$$

for any $x$ and $y$ in $A$. Thus $B$ and $C$, the matrices of coefficients given above, clearly satisfy relations (2.2). Conversely, relations (2.2) furnish an $A$-module, namely the module of all $n$ tuples, with bases of length $n$ and $n+k$.

Call a mapping from $A$ to $A^{\prime}$ a rurap mapping if right units are mapped into right units and if the image of a right annihilator is also a right annihilator. (A similar mapping with right changed to left everywhere is called a lulap mapping.) We remark that any onto mapping is a rurap mapping. Using the partial ordering $0<(n, k)<d$ and $\left(n^{\prime}, k^{\prime}\right) \leqq(n, k)$ if and only if $n^{\prime} \leqq n$ and $k^{\prime} \mid k$, we may prove

THEOREM 2.4. If $A \rightarrow A^{\prime}$ is a rurap homomorphism, then $t\left(A^{\prime}\right) \leqq t(A)$.

Proof. If $A$ is a zero ring, so is $A^{\prime}$. Thus the theorem is clear if either $t(A)=0$ or $t\left(A^{\prime}\right)=0$, and is also clear if $t(A)=d$. The case $t\left(A^{\prime}\right)=d$ will be settled in what is to follow. Thus let $t\left(A^{\prime}\right)=\left(n^{\prime}, k^{\prime}\right)$. By Theorem 2.3, if $t(A)=(n, k)$, there exist matrices $B$ and $C$ satisfying (2.2) whose images $B^{\prime}$ and $C^{\prime}$ under the rurap homomorphism $A \rightarrow A^{\prime}$ also satisfy (2.2). Hence, by Theorem 2.3, $t\left(A^{\prime}\right)=d$ implies $t(A)=d$. It also follows, when $t(A)=(n, k)$, that $A^{\prime}$ admits a module with bases of length $n$ and $n+k$. Thus, by Theorem 2.2, $n^{\prime} \leqq n$ and, for some $h$, $n=h+m_{1} k^{\prime}$ and $n+k=h+m_{2} k^{\prime}$. Hence $k^{\prime} \mid k$ and so $\left(n^{\prime}, k^{\prime}\right) \leqq(n, k)$.

The following theorem shows that there is a close connection between the concepts of type and the module type of [2]. 
THEOREM 2.5. Let $A$ be a ring with right units; then there exists a homomorphic image $\bar{A}$ of $A$, with unit, such that the module type of $\bar{A}=t(A)$.

Proof. Let $e$ be a right unit of $A$. The set $J=\{e x-x: x \in A\}$ is a two-sided ideal of $A$. If $J=0$, then $e$ is the unique two-sided unit of $A$, and we can choose $\bar{A}=A$. Thus assume that $A$ has no two-sided unit, so that there exists a right unit $f(f \neq e)$ in $A$. If $J=A$, then $e x-x=f$ for some $x \in A$, and, for all $z \in A, z=z f=z(e x-x)=0$; a contradiction. Hence $J \neq A$. This shows that $A / J$ is a non-zero ring with unit $\bar{e}=e+J$. Let $A / J=\bar{A}$. Since $A \rightarrow \bar{A}$ is a rurap homomorphism (being an onto mapping), by Theorem 2.4,

$$
\text { module type of } \bar{A}=t(\bar{A}) \leqq t(A) \text {. }
$$

Thus, if the module type of $\bar{A}=d$, we have $t(A)=d$. We remark that, by the above, if $A \neq 0$, then $\bar{A} \neq 0$. Thus the module type of $\bar{A}=0$ if and only if $t(A)=0$.

Assume that the module type of $\bar{A}=(n, k)$. Then, by the matrix criterion in [2, p. 115], there exist $n$ by $n+k$ and $n+k$ by $n$ matrices $\bar{B}$ and $\bar{C}$ over $\bar{A}$ such that $\bar{B} \bar{C}-\bar{I}_{n}=0$ and $\bar{C} \bar{B}-\bar{I}_{n+k}=0$, where $\bar{I}_{n}$ and $\bar{I}_{n+k}$ are identity matrices over $\bar{A}$. Thus, if $B \rightarrow \bar{B}$ and $C \rightarrow \bar{C}$, then $B C-I_{n} \in J_{n}$ and $C B-I_{n+k} \in J_{n+k}$, where $I_{n}$ and $I_{n+k}$ are diagonal matrices with $e$ the diagonal entry, while $J_{n}$ and $J_{n+k}$ are matrices over $J$. But since $A J=0$, it follows that $X B C=X I_{n}=X$ and $Y C B=Y I_{n+k}=Y$ for any $n$-dimensional vector $X$ and $(n+k)$-dimensional vector $Y$. Hence, by Theorem $2.3, t(A) \leqq(n, k)$ and thus equation (2.3) implies that $t(A)=$ module type of $\vec{A}$.

By virtue of this theorem all results of [2] apply immediately to rings with right units.

3. The maxit of a ring. Throughout this section and the next section, rings will not necessarily possess one-sided units. We shall also assume that any ring considered has at least one homomorphic image which is a non-zero ring with right units. Let $A$ be a ring and $\alpha_{i}: A \rightarrow A_{t}$ ( $i \in I$, an index set) be homomorphisms from $A$ onto rings with right units $A_{i}$. By the results in the previous section, $t\left(A_{i}\right)$ is meaningful. Let $\mathscr{I}(A)$ represent the set of all such homomorphic images of $A$. With $\mathscr{I}(A)$ we associate the set $\left\{t\left(A_{i}\right)\right\}$ and we let

$$
M(A)=\bigcup_{A_{i} \in \mathscr{I}(A)} t\left(A_{i}\right) \quad\left[\equiv \sup _{A_{i} \in \mathscr{I}(A)} t\left(A_{i}\right)\right] .
$$

We note that, if all $t\left(A_{i}\right)=0$, then $M(A)=0$; if all $t\left(A_{i}\right)=\left(n_{i}, k_{i}\right)$ or 0 and if $\max \left\{n_{i}\right\}$ and l.c.m. $\left\{k_{i}\right\}$ exist, then $M(A)=(n, k)=\left(\max \left\{n_{i}\right\}\right.$, l.c.m. $\left.\left\{k_{i}\right\}\right)$; otherwise $M(A)=d$. We shall call $M(A)$ the maximal image type of $A$, abbreviated as the maxit of $(A)$.

If $A$ has right units, then, since $\alpha_{i}: A \rightarrow A_{i}$ are onto homomorphisms, they are rurap homomorphisms and, by Theorem $2.4, t\left(A_{i}\right) \leqq t(A)$. Since $A \in \mathscr{I}(A)$, we have $M(A)=t(A)$. Thus for rings with right units the concept of maxit and type are the same. Hence this is a proper generalization of module type of a ring defined in [2]. Also note that we can use the same partial ordering for maxits as for types (see preceding section).

THEOREM 3.1. If $A \rightarrow A^{\prime}$ is an onto homomorphism, then $M\left(A^{\prime}\right) \leqq M(A)$.

Proof. If $M(A)=d$ or $M\left(A^{\prime}\right)=0$, the theorem is trivial. If $A_{i}^{\prime} \in \mathscr{I}\left(A^{\prime}\right)$, then $A_{i}^{\prime} \in \mathscr{I}(A)$. Hence $\mathscr{I}\left(A^{\prime}\right) \subseteq \mathscr{I}(A)$. But $M\left(A^{\prime}\right)=\mathrm{U}_{t}\left(A_{i}\right)$ over all $A_{i} \in \mathscr{I}\left(A^{\prime}\right)$ and $M(A)=\mathrm{U}_{t}\left(A_{i}\right)$ over all $A_{i} \in \mathscr{I}(A)$. Hence $M\left(A^{\prime}\right) \leqq M(A)$. 
COROLlaRY 1. $M(A)=d$ if and only if there exists an onto homomorphism from $A$ to a ring $A^{\prime}$ such that $M\left(A^{\prime}\right)=d$.

COROLlary 2. Let $A$ be a commutative ring; then $M(A)=d$.

Proof. Let $I$ be a maximal modular ideal of $A$. Then $A / I$ is a field, and $A \rightarrow A / I$ is an onto homomorphism.

Corollary 3. Let $\left\{x_{i}\right\}$ ( $i \in I$, an index set) be a set of symbols, $B$ the polynomial ring in symbols $\left\{x_{i}\right\}$ (commuting or not) over $A$; then $M(A) \leqq M(B)$.

Proof. Map polynomials to their constant term.

We remark that the maxits form a distributive lattice using the partial ordering defined above and defining $U$ and $n$ as follows:

and

$$
\begin{aligned}
& (n, k) \cup\left(n^{\prime}, k^{\prime}\right)=\left(\max \left\{n, n^{\prime}\right\}, \text { l.c.m. }\left\{k, k^{\prime}\right\}\right), \\
& (n, k) \cup d=d, \quad(n, k) \cup 0=(n, k) ;
\end{aligned}
$$

$$
\begin{aligned}
& (n, k) \cap\left(n^{\prime}, k^{\prime}\right)=\left(\min \left\{n, n^{\prime}\right\}, \text { g.c.d. }\left\{k, k^{\prime}\right\}\right), \\
& (n, k) \cap d=(n, k), \quad(n, k) \cap 0=0 .
\end{aligned}
$$

ThEOREM 3.2. If $A=A_{1} \oplus A_{2}$ is a ring direct sum, then $M(A)=M\left(A_{1}\right) \cup M\left(A_{2}\right)$.

Proof. The projections $A \rightarrow A_{i}(i=1,2)$ are onto homomorphisms, and so, by Theorem 3.1, $M\left(A_{i}\right) \leqq M(A)(i=1,2)$. Therefore, if either $M\left(A_{i}\right)$ or $M\left(A_{2}\right)$ equals $d$, then

$$
M\left(A_{1}\right) \cup M\left(A_{2}\right)=d \text { and } M(A)=d .
$$

Also, if either $M\left(A_{i}\right)=0$, say $M\left(A_{1}\right)=0$, then $M(A)=M\left(A_{2}\right)$. Let $\alpha: A \rightarrow B$ be an onto homomorphism with $B \in \mathscr{I}(A)$. The restriction of $\alpha$ to $A_{i}(i=1,2)$ induces onto homomorphisms $\alpha_{i}$ onto $B_{i}$ (i.e. $B_{i}=A_{i} \alpha$ ) and $B=B_{1} \oplus B_{2}$ is a ring direct sum. $B$ is a ring with right units and, by Theorem 3 of $\left[2\right.$, p. 116], $t(B)=t\left(B_{1}\right) \cup t\left(B_{2}\right)$. Hence for $B^{j} \in \mathscr{I}(A)$ we have $t\left(B^{j}\right)=t\left(B_{1}^{j}\right) \cup t\left(B_{2}^{j}\right)$, where $B^{j}=B_{1}^{j} \oplus B_{2}^{j}$. Hence

$$
M(A)=\bigcup_{B^{\prime} \in \mathcal{F}(A)}^{\bigcup} t\left(B^{j}\right)=\left[\bigcup_{t}\left(B_{1}^{j}\right)\right] \cup\left[\bigcup_{t}\left(B_{2}^{j}\right)\right]=M\left(A_{1}\right) \cup M\left(A_{2}\right) .
$$

Let $\left\{k_{l}\right\}$ be a (possibly infinite) set of positive integers and let $m$ be a fixed integer. Define

$$
k=\text { I.c.m. }\left\{k_{i}\right\}, \quad g_{i}=\text { g.c.d. }\left(k_{i}, m\right), \quad g=\text { g.c.d. }(k, m), \quad k^{\prime}=1 . \text {.m. }\left\{k_{i}\right\},
$$

assuming that these exist.

Then we can prove

LEMMA 3.1. The following relations hold for the above integers:

$$
\text { (i) } k / g=1 . \mathrm{c} . \mathrm{m} .\left\{k_{i} / g_{i}\right\} \text { and (ii) } k^{\prime} / g=\text { g.c.d. }\left\{k_{i} / g_{i}\right\} \text {. }
$$


Proof. We shall prove (i); (ii) can be proved similarly. We shall use the prime decompositions

Then

$$
k_{i}=\prod p_{j}^{a_{i j}} \quad\left(a_{i j} \geqq 0\right) ; \quad m=\prod p_{j}^{b_{j}} \quad\left(b_{j} \geqq 0\right) .
$$

$$
\begin{aligned}
& k=\prod p_{j}^{\max \left\{a_{i j}\right\}}, \quad g_{i}=\prod p_{j}^{\min \left(a_{i j}, b_{j}\right)}, \quad k_{i} / g_{i}=\prod p_{j}^{a_{i j}-\min \left(a_{i j}, b_{j}\right)}, \\
& g=\prod p_{j}^{\min \left(\max \left\{a_{i j}\right\}, b_{j}\right)}, \quad k / g=\prod p_{j}^{\max \left\{a_{i j}\right\}-\min \left(\max \left\{a_{i j}\right\}, b_{j}\right)} .
\end{aligned}
$$

We have to show that, for each prime $p_{j}$,

$$
\max \left\{a_{i j}\right\}-\min \left(\max \left\{a_{i j}\right\}, b_{j}\right)=\max \left\{a_{i j}-\min \left(a_{i j}, b_{j}\right)\right\} .
$$

Let $a_{s j}=\max \left\{a_{i j}\right\}$. If $a_{s j} \geqq b_{j}$, then the left- and right-hand sides of (3.1) are equal to $a_{s j}-b_{j}$. If $b_{j} \geqq a_{s j}$, then both sides are zero.

TheOREM 3.3. Let $A_{m}$ be the complete matrix ring over $A$. Then

(i) $M\left(A_{m}\right)=d$ if $M(A)=d$ and (ii) if $M(A)=(n, k), M\left(A_{m}\right) \geqq((n+r) / m, k / g)$, where $r$ is the least non-negative integer such that $m \mid(n+r)$ and $g=$ g.c.d. $(k, m)$.

Proof. The homomorphisms $\alpha_{i}: A \rightarrow A_{i}\left(A_{i} \in \mathscr{I}(A)\right)$ induce homomorphisms from $A_{m}$ onto $\left(A_{i}\right)_{m}$, the matrix ring over $A_{i}$. Hence, by Theorem 3.1,

$$
M\left[\left(A_{i}\right)_{m}\right] \leqq M\left(A_{m}\right) \text {. }
$$

Since $A_{i}$ is a ring with right units, $M\left(A_{i}\right)=t\left(A_{i}\right)$. Thus if $t\left(A_{i}\right)=\left(n_{i}, k_{i}\right)$, then by [2, Theorem 5, p. 116], $M\left[\left(A_{i}\right)_{m}\right]=\left(\left(n_{i}+r_{i}\right) / m, k_{i} / g_{i}\right)$, where $r_{i}$ is the least non-negative integer such that $m \mid\left(n_{i}+r_{i}\right)$ and $g_{i}=$ g.c.d. $\left(k_{i}, m_{i}\right)$. If $n=\max \left\{n_{i}\right\}$ exists, then $n+r \geqq n_{i}+r_{i}$ for all $i$ and for some $r \geqq 0$; so $(n+r) / m=\max \left\{\left(n_{i}+r_{i}\right) / m\right\}$. Also, if $k=1$.c.m. $\left\{k_{i}\right\}$ exists, then, by Lemma 3.1, 1.c.m. $\left\{k_{i} / g_{i}\right\}=k / g$, where $g_{i}$ and $g$ are defined as in the lemma. But

and by (3.2)

$$
\bigcup M\left[\left(A_{i}\right)_{m}\right]=\bigcup_{i}\left(\left(n_{i}+r_{i}\right) / m, k_{i} / g_{i}\right)=((n+r) / m, k / g)
$$

$$
((n+r) / m, k / g) \leqq M\left[\left(A_{m}\right)\right] .
$$

Now, if $M(A)=d$, because $t\left(A_{i}\right)=d$ for some $A_{i} \in \mathscr{I}(A)$, then $M\left[\left(A_{i}\right)_{m}\right]=d$, and thus, by (3.2), $M\left(A_{m}\right)=d$. Also, if either $\max \left\{n_{i}\right\}$ or l.c.m. $\left\{k_{i}\right\}$ do not exist, then either $\max \left(\left(n_{i}+r_{i}\right) / m\right\}$ or l.c.m. $\left\{k_{i} / g_{i}\right\}$ fails to exist and again $d \leqq M\left(A_{m}\right)$; so $M\left(A_{n}\right)=d$.

THEOREM 3.4. If $A$ is Noetherian (Artinian), then $M(A)=d$.

Proof. For $A_{i} \in \mathscr{I}(A), A_{i}$ is also Noetherian (Artinian) and hence $\left[2\right.$, p. 114] $t\left(A_{i}\right)=d$. Thus $M(A)=d$.

4. The minit of a ring. In this section we shall define a concept " dual " to the one defined in $\S 3$. Let $\alpha_{i}: A \rightarrow A_{i}$ be homomorphisms from $A$ onto non-zero rings with right units (assuming that $A$ is not the zero ring). As before, let $\mathscr{I}(A)$ denote the set of all such homomorphic images of $A$. Define

$$
m(A)=\bigcap_{A_{i} \in \mathcal{S}_{(A)}} t\left(A_{i}\right)\left[\equiv \inf _{A_{i} \in \mathcal{F}_{(A)}} t\left(A_{i}\right)\right]
$$


and call $m(A)$ the minimal image type of $A$ (abbreviated the minit). Note that $m(A)=d$ if and only if all $t\left(A_{i}\right)=d$. If any $t\left(A_{i}\right)=\left(n_{i}, k_{i}\right)$, then, since no member of $\mathscr{I}(A)$ is zero,

$$
m(A)=\left(\min \left\{n_{i}\right\}, \text { g.c.d. }\left\{k_{i}\right\}\right) .
$$

In the case which $A$ is the zero ring, define $m(A)=0$.

If $A$ is itself a ring with right units, then $A \in \mathscr{I}(A)$. Hence $m(A) \leqq t(A)$. Equality can hold, as for example in the case of a commutative ring whose minit will be shown (see Theorem 4.2 ) to be $d$. That strict inequality is also possible is seen from the example constructed in [2, p. 118]. In this example, $R$ is a ring of polynomials (free algebra) and so by [2, Corollary 4 , p. 116] $t(R)=d$. But it is shown [2, pp. 118-130] that there exists an ideal $H$ such that $t(R / H)=(1, k)$ for any $k \geqq 2$. Since $R / H \in \mathscr{J}(A)$, we have $m(R) \leqq(1, k)<d$.

We remark that under the same partial ordering as for types and maxits, and with the same definitions of $n$ and $\cup$, the minits also form a distributive lattice.

THEOREM 4.1. If $A \rightarrow A^{\prime}$ is an onto homomorphism, then $m(A) \leqq m\left(A^{\prime}\right)$.

Proof. This is clear since $\mathscr{I}\left(A^{\prime}\right) \subseteq \mathscr{I}(A)$.

COROLLARY 1. $m(A)=d$ if and only if $A$ is the homomorphic image of a ring with minit $d$.

COROLlaRY 2. Let $\left\{x_{i}\right\}$ be a set of symbols and $B$ the ring of polynomials in $\left\{x_{i}\right\}$ (commuting or not) over $A$. Then $m(B) \leqq m(A)$.

Proof. Map polynomials to their constant terms.

We remark that for the example mentioned above $m(R)<m\left(Z_{2}\right)=d$ (where $Z_{2}$ is the field of integers modulo 2); so strict inequality can hold.

THEOREM 4.2. Let $A$ be a commutative ring; then $m(A)=d$.

Proof. If $A_{i} \in \mathscr{I}(A)$, then $A_{i}$ is a commutative ring with right units and hence [2, Corollary 3, p. 115] $t\left(A_{i}\right)=d$. Thus $m(A)=d$.

THeOREM 4.3. Let $A=A_{1} \oplus A_{2}$, the ring direct sum; then $m(A)=m\left(A_{i}\right) \cap m\left(A_{2}\right)$.

Proof. The projection mappings from $A$ to $A_{i}(i=1,2)$ are onto homomorphisms. By Theorem 4.1, $m(A) \leqq m\left(A_{i}\right)(i=1,2)$. Hence $m(A) \leqq m\left(A_{1}\right) \cap m\left(A_{2}\right)$. Now suppose $\alpha: A \rightarrow B$ such that $B \in \mathscr{I}(A)$; then $B=B_{1} \oplus B_{2}$, where $B_{i}=A_{i} \alpha$. Thus by [2, Theorem 3, p. 115] $t(B)=t\left(B_{1}\right) \cup t\left(B_{2}\right)$. Hence for $B \in \mathscr{I}(A)$, there exists an image $B_{1}$ of $A_{1}$ such that $t\left(B_{1}\right) \leqq t(B)$. Hence $m\left(A_{1}\right) \leqq m(A)$ and similarly $m\left(A_{2}\right) \leqq m(A)$. Thus $m\left(A_{1}\right) \cap m\left(A_{2}\right) \leqq m(A)$. It follows that $m(A)=m\left(A_{1}\right) \cap m\left(A_{2}\right)$.

It is an open question whether an algebraic construction exists by which, from two given rings $A_{1}, A_{2}$, a ring may be obtained whose minit equals $m\left(A_{1}\right) \cup m\left(A_{2}\right)$.

THEOREM 4.4. Let $A_{1}$ and $A_{2}$ be rings all of whose homomorphic images are rings with right units, and let $A=A_{1} \otimes A_{2}$ be their tensor product. Then $m(A) \leqq m\left(A_{1}\right) \cap m\left(A_{2}\right)$.

Proof. It is easily shown that $B \in \mathscr{I}(A)$ if and only if $B=B_{1} \otimes B_{2}$, where $B_{1} \in \mathscr{I}\left(A_{1}\right)$ and $B_{2} \in \mathscr{I}\left(A_{2}\right)$. Since [2, Theorem 4, p. 116] $t(B) \leqq t\left(B_{1}\right) \cap t\left(B_{2}\right)$ for all such rings $B$, it is clear that $m(A) \leqq m\left(A_{1}\right) \cap m\left(A_{2}\right)$. 
THEOREM 4.5. Let $A_{s}$ be the complete matrix ring over $A$; then (i) $m\left(A_{s}\right)=d$ implies $m(A)=d$, and (ii) if $m(A)=(n, k)$, then $m\left(A_{s}\right) \leqq((n+r) / s, k / g)$, where $r$ is the least non-negative integer such that $m \mid(n+r)$ and $g=$ g.c.d. $(k, s)$.

Proof. Let $m(A)=(n, k)$; then there exist onto homomorphisms $\alpha_{i}: A \rightarrow A_{i}$ such that $t\left(A_{i}\right)=\left(n_{i}, k_{i}\right)$ and $m(A)=\bigcap t\left(A_{i}\right)$. The homomorphisms $\alpha_{i}$ induce homomorphisms from $A_{s}$ onto $\left(A_{i}\right)_{s}$. Hence, by Theorem $4.1, m\left(A_{s}\right) \leqq m\left[\left(A_{i}\right)_{s}\right]$. Since $\left(A_{i}\right)_{s}$ is a ring with right units,

$$
m\left[\left(A_{i}\right)_{s}\right] \leqq t\left[\left(A_{i}\right)_{s}\right]=\left(\left(n_{i}+r_{i}\right) / s, k_{i} / g_{i}\right),
$$

where $r_{i}$ is the least non-negative integer such that $s \mid\left(n_{i}+r_{i}\right)$ and $g_{i}=$ g.c.d. $\left(k_{i}, s\right)$. Since $n=\min \left\{n_{i}\right\}, n+r \leqq n_{i}+r_{i}$ for all $i$. Hence $\min \left\{\left(n_{i}+r_{i}\right) / s\right\}=\{(n+r) / s\}$. By Lemma 3.1, g.c.d. $\left\{k_{i} / g_{i}\right\}=k / g$. Hence $m\left(A_{s}\right) \leqq \bigcap m\left[\left(A_{i}\right)_{s}\right] \leqq \bigcap\left(\left(n_{i}+r_{i}\right) / s, k_{i} / g_{i}\right)=((n+r) / s, k / g)$.

Now if $m\left(A_{s}\right)=d$, then all $t\left[\left(A_{i}\right)_{s}\right]=d$ and, by [2, Theorem 5, p. 117], $t\left(A_{i}\right)=d$. Thus $m(A)=d$.

THEOREM 4.6. If $A$ is a Noetherian (Artinian), then $m(A)=d$.

Proof. $A_{i} \in \mathscr{I}(A)$ implies that $A_{i}$ is Noetherian (Artinian). Hence all $t\left(A_{i}\right)=d$ and $m(A)=d$.

5. Applications. A ring $A$ is called strongly dimensional if $t(A)=M(A)=m(A)=d$. (i.e. if all its homomorphic images have type $d$.) In $\S 4$ we gave an example, the ring $R$, such that $t(R)=d$ but $m(R)<d$. Hence there are dimensional rings that are not strongly dimensional. Commutative rings, Noetherian and Artinian rings are examples of strongly dimensional rings.

A ring $A$ possessing property $P$ is said to be homomorphically $P$ if all non-zero homomorphic images of $A$ have property $P$. Every Noetherian ring is homomorphically-Noetherian, but there are semisimple rings (for example the integers) which are semisimple but not homomorphically-semisimple.

LEMMA 5.1. A Jacobson semi-primary ring $[3$, p. 56] is homomorphically semi-primary.

Proof. Let $\alpha: A \rightarrow A^{\prime}$ be an onto homomorphism and $J(A), J\left(A^{\prime}\right)$, the respective Jacobson radicals of $A, A^{\prime}$. Clearly $J(A) \alpha \subseteq J\left(A^{\prime}\right)$ and hence $\alpha$ induces the mapping $A / J(A) \rightarrow A^{\prime} \mid J\left(A^{\prime}\right)$. Thus $A / J(A)$ Artinian implies $A^{\prime} / J\left(A^{\prime}\right)$ Artinian; so $A^{\prime}$ is semi-primary.

THEOREM 5.1. A semi-primary ring $A$ is strongly dimensional.

Proof. Let $A^{\prime} \in \mathscr{I}(A)$. Then, by Lemma $5.1, A^{\prime}$ is a semi-primary ring with right units. But $A^{\prime} \mid J\left(A^{\prime}\right)$ is Artinian; hence $t\left(A^{\prime} \mid J\left(A^{\prime}\right)\right)=d . A^{\prime} \rightarrow A^{\prime} \mid J\left(A^{\prime}\right)$ is an onto homomorphism; so, by Theorem $2.4, t\left(A^{\prime}\right)=d$. Therefore $m(A)=M(A)=t(A)=d$.

COROLLARY 1. Jacobson primary rings and completely primary rings $[3, \mathrm{p} .56]$ are strongly dimensional.

R. E. Blair in [1] examined a ring satisfying condition $D_{r}$; its lattice of right ideals is distributive. 
Lemma (Blair) A non-zero semisimple ring that satisfies $D_{r}$ is isomorphic to a subdirect sum of division rings.

Proof. See [1, p. 148].

THEOREM 5.2. A semisimple ring that is homomorphically semisimple and satisfies $D_{r}$ is strongly dimensional.

Proof. By Blair's lemma $A=\Sigma \dot{+} D_{i}$, a subdirect sum of division rings. Hence there exist ideals $I_{i}$ in $A$ (with zero intersection) such that $A / I_{i}$ is isomorphic to $D_{i}$. Since $t\left(D_{i}\right)=d$, $t\left(A / I_{i}\right)=d$. The natural homomorphism $A \rightarrow A / I_{i}$ is onto, and so, by Theorem $2.4, t(A)=d$. If $A^{\prime} \in \mathscr{I}(A)$, then $A^{\prime}$ is semisimple by hypothesis. Also $A^{\prime}$ satisfies $D_{r}$, since if $B$ is an ideal of $A$, the sublattice of right ideals between $A$ and $B$ is distributive and clearly order isomorphic to the right ideal lattice of $A / B$. Thus $t\left(A^{\prime}\right)=d$, and $M(A)=m(A)=t(A)=d$.

Note that from the above proof $t(A)=d$ for any semisimple ring satisfying $D_{r}$.

Other rings which are easily shown to be strongly dimensional include (i) simple rings with unit having minimal one-sided ideals, (ii) regular (von Neumann) rings with non-zero nilpotents, and (iii) local rings.

\section{REFERENCES}

1. R. E. Blair, Ideal lattices and the structure of a ring, Trans. Amer. Math. Soc. 75 (1953), 136-153.

2. W. G. Leavitt, The module type of a ring, Trans. Amer. Math. Soc. 103 (1962), 113-130.

3. N. Jacobson, Structure of rings, American Mathematical Society Colloquium Publications, Vol. 37 (Providence, R. I., 1956).

UNIVERSITY OF IOWA and UNIVERSITY OF NEBRASKA 